\title{
Development and validation of a new two-dimensional wake model for wind turbine wakes
}

Tian, Linlin; Zhu, Wei Jun; Shen, Wen Zhong; Zhao, Ning; Shen, Zhiwei

Published in:

Journal of Wind Engineering and Industrial Aerodynamics

Link to article, DOI:

10.1016/j.jweia.2014.12.001

Publication date:

2015

Document Version

Publisher's PDF, also known as Version of record

Link back to DTU Orbit

Citation (APA):

Tian, L., Zhu, W. J., Shen, W. Z., Zhao, N., \& Shen, Z. (2015). Development and validation of a new twodimensional wake model for wind turbine wakes. Journal of Wind Engineering and Industrial Aerodynamics, 137, 90-99. https://doi.org/10.1016/j.jweia.2014.12.001

\section{General rights}

Copyright and moral rights for the publications made accessible in the public portal are retained by the authors and/or other copyright owners and it is a condition of accessing publications that users recognise and abide by the legal requirements associated with these rights.

- Users may download and print one copy of any publication from the public portal for the purpose of private study or research.

- You may not further distribute the material or use it for any profit-making activity or commercial gain

- You may freely distribute the URL identifying the publication in the public portal 


\title{
Development and validation of a new two-dimensional wake model for wind turbine wakes
}

\author{
Linlin Tian ${ }^{\text {a,* }}$, Weijun Zhu ${ }^{\text {b }}$, Wenzhong Shen ${ }^{\mathrm{b}}$, Ning Zhao ${ }^{\mathrm{a}}$, Zhiwei Shen ${ }^{\mathrm{a}}$ \\ a Jiangsu Key Laboratory of Hi-Tech Research for Wind Turbine Design, Nanjing University of Aeronautics and Astronautics, Nanjing, Jiangsu, \\ P.R.China, 210016 \\ ${ }^{\mathrm{b}}$ Department of Wind Energy, Fluid Mechanics Section, Building 403, Technical University of Denmark, Lyngby, Denmark, DK-2800
}

\section{A R T I C L E I N F O}

\section{Article history:}

Received 11 July 2014

Received in revised form

6 December 2014

Accepted 6 December 2014

\section{Keywords:}

Jensen wake model

Wind turbine wakes

Velocity deficit

Variable wake decay constant

Wind farm development projects

\begin{abstract}
A B S T R A C T
A new two-dimensional (2D) wake model is developed and validated in this article to predict the velocity and turbulence distribution in the wake of a wind turbine. Based on the classical Jensen wake model, this model is further employing a cosine shape function to redistribute the spread of the wake deficit in the crosswind direction. Moreover, a variable wake decay rate is proposed to take into account both the ambient turbulence and the rotor generated turbulence, different from a constant wake decay rate used in the Jensen model. The obtained results are compared to field measurements, wind tunnel experiments, and results of an advanced $k-\omega$ turbulence model as well as large eddy simulations. From the comparisons, it is found that the proposed new wake model gives a good prediction in terms of both shape and velocity amplitude of the wake deficit, especially in the far wake which is the region of interest for wind farm development projects.
\end{abstract}

(c) 2014 Elsevier Ltd. All rights reserved.

\section{Introduction}

The Jensen wake model (Jensen, 1983; Katic et al., 1986), also named as the PARK wake model, is one of the most widely used analytical wake models, which has been implemented in commercial codes such as WAsP, GH WindFarmer, WindPRO and OpenWind. Based on the law of global momentum conservation, the Jensen model provides a mathematical expression of the wake velocity deficit to describe the wake of a single wind turbine. In this model, the wake width is assumed to linearly expand downstream with a wake decay constant $(k)$, which is an empirically determined parameter. The suggested value of $k$ in the literature (Barthelmie et al., 2006) is 0.075 for onshore cases and 0.05 for offshore applications. The Jensen model is easy to code with only a few input parameters. Despite its simplicity, the Jensen model has been proved to provide an acceptable representation of the wake behavior (Crespo et al., 1999; Porte Agel et al., 2013; Schlez et al., 2003). For single wake prediction, the results predicted by the Jensen model (Magnusson and Smedman, 1999) are in reasonable agreement with available data. Barthelmie et al. (2009) evaluated the Jensen model and five other engineering models against a set of

\footnotetext{
* Corresponding author.

E-mail addresses: blusius@126.com (L. Tian),wjzh@dtu.dk (W. Zhu), wzsh@dtu.dk (W. Shen), zhaoam@nuaa.edu.cn (N. Zhao), shenzhiweitc@163.com (Z. Shen).
}

experiments from an offshore wind farm. In addition, a comparison of different wake models (Barthelmie et al., 2006) shows that there is no particular difference between the analytical models and sophisticated models in term of accuracy. All these tests and validations have proved that even the Jensen model is old and simple, it can still exhibit a good, yet not perfect, match with the measured data.

The Jensen wake model is a one-dimensional (1D) model because the wake speed is assumed to be the only variable in function of the downwind distance, meaning that the predicted wake velocity profile along the crosswind direction at a certain downstream position is assumed to be uniform. On the other hand, the theoretical knowledge and field measurements (Vermeer et al., 2003) have pointed out that the assumption of the top-hat distribution in the 1D Jensen model is not realistic. In addition, it is also claimed by Katic et al. (1986) that the Jensen model gives an estimation of the output energy rather than describing the velocity field accurately due to the top-hat assumption.

In this article, a two-dimensional (2D) wake model (named as 2D Jensen model in this article) is presented by distributing the velocity profile in the cross section with a cosine shape instead of the top-hat shape in the standard Jensen model. Moreover, by taking into account the effect of turbulence on the wake recovery, the wake decay parameter $k$ becomes a variable depending on both the atmospheric and the rotor generated turbulence, and also the downstream distance from the wind turbine. For this 
purpose, an analytical model for estimating the added turbulence generated by the presence of the wind turbine is proposed at first and then compared with some other engineering models to assess its validity.

The structure of this article is organized as follows: In Section 2, a brief review of the Jensen model is presented. After that, the 2D Jensen model is derived in Section 3. The developed model is then applied in several test cases to validate its accuracy and generality, including different types of wind turbines under different operating conditions and various atmospheric turbulence levels. Results are presented and compared with wind tunnel measurements, field measurements as well as numerical results from an advanced CFD (Computational Fluid Dynamics) method in Section 4. Finally conclusions and perspectives are given in Section 5.

\section{Standard Jensen wake model}

To derive the $2 \mathrm{D}$ Jensen model, a brief introduction of the $1 \mathrm{D}$ Jensen model is necessary. The Jensen model is based on the assumption that the wake velocity at a given downwind position can be expressed in terms of turbine's thrust coefficient $C_{T}$ and a semi-empirical wake decay constant $k$

$u^{*}=u_{0}\left[1-2 a /\left(1+k x / r_{1}\right)^{2}\right]$

where $u^{*}$ is the wake velocity at the downstream position $x, u_{0}$ is the incoming wind speed, $a$ is the axial induction factor calculated from the thrust coefficient $C_{T}$ of the wind turbine using the following formula

$a=\left(1-\sqrt{1-C_{T}}\right) / 2$

and $r_{1}$ is the characteristic downstream rotor radius represents the expanded wake radius immediately downstream of the wind turbine, which can be computed using

$r_{1}=r_{d} \sqrt{(1-a) /(1-2 a)}$

where $r_{d}$ is the rotor radius of the wind turbine.

The Jensen model assumes that the wake expands linearly with a parameter wake decay constant $k$ denoting the growth of the wake width per unit length in the downwind direction. The expressions for $k$ and the spread wake radius $r_{x}$ (the wake radius denotes the distance between the rotor axis and the first point where the wind speed is equal to the free stream value) are given as follows

$k=0.5 / \ln \left(z / z_{0}\right) ; \quad r_{x}=k x+r_{d}$

where $z$ is the hub height of the wind turbine, $z_{0}$ is the surface roughness height of a local terrain.

When considering the wake flow area and the outward free stream area at a certain downstream position, it is found that the shape of the wake velocity along the cross-stream direction is like a top-hat, therefore the distribution of predicted wake velocity by the $1 \mathrm{D}$ Jensen model is also called a top-hat distribution. It should be mentioned that the Jensen wake model assumes the wake is fully turbulent and the contribution from the tip vortices is negligible, which leads to the fact that the Jensen model is not designed for use in the near-wake condition. It should be applicable to capture the velocity deficit for a downstream distance in excess of three rotor diameters (3D) (Barthelmie et al., 2006).

\section{2D Jensen wake models}

In this section, the new 2D wake model is presented. The wake velocity distribution model is first introduced in Section 3.1. Then the wake turbulence distribution model is developed in Section 3.2 to take the turbulence into account in the wake model. In the last Section 3.3, the 2D wake model with turbulence is proposed based on the 2D Jensen model described in Section 3.1 and the wake decay parameter in the function of the wake turbulence developed in Section 3.2. Note that for the sake of simplicity, this model is called 2D_k wake model in this work.

\subsection{The proposal of a $2 D$ Jensen wake model}

By letting the wake deficit only vary with the downstream distance $x$, the Jensen wake model is referred to as a onedimensional model. However, according to the classical theories of shear flows in the wakes of bluff bodies (Dufresne and Wosnik, 2013) as well as the wind tunnel investigations for wake behind a single wind turbine (Chamorro and Porte-Agel, 2009), it is found that the velocity in the wake has an approximately Gaussian axisymmetric shape after a certain downstream distance. To carry out more detailed investigation of wind turbine wake, the standard Jensen model seems too simple because it only describes the center line wake deficit in the cross-stream section. Thus a proper modification is highly needed to represent the physical wake distribution.

In most cases, turbine spacing at operational offshore wind farm is currently in the range of 4D-12D (Barthelmie et al., 2010). So a good engineering wake model should have the ability to accurately predict the wake development in this range. In the work of (RÉTHORÉ, 2006), it is pointed out that the Gaussian distribution which based on the self-similar theory showed a quite large error at the near wake region, and it seems to be acceptable after six rotor diameters. But in the study by N.O. Jensen (Jensen, 1983), the author validated a cosineshape profile of the velocity deficit in the cross-wind direction against the measurements from the Nibe wake project. Agreement between the numerical prediction and the measurements was seen to be satisfactory at both the near wake and far wake areas. Besides, in the reference (Taylor, 1990) it is mentioned that at the far wake region the velocity deficit is supposed to have adopted a self-preserving form, which is usually represented either by a Gaussian or a closely similar polynomial relation. According to these, we try to use the similar sinusoidal function to fit the profile of the wake deficit. So the simple cosine function is employed here instead of a Gaussian distribution, and hence a 2D wake velocity model is developed. The equation of the new model is defined as

$u=A \cos (K \times r+\pi)+B$

of which, $A, K$ and $B$ are determined constants, $r$ is the radial distance from the center of the wake. For the sake of simplicity, this 2D wake model is called 2D Jensen model because it is based on the original Jensen wake model and the following assumptions:

- Assumption 1: The new model has the same wake radius as the original model

$\frac{2 \pi}{K}=2 r_{x}$

- Assumption 2: When the variable $r$ tends to the outer boundary of the wake region $r_{x}$, the mean wind speed reaches the freestream wind speed

$A \cos \left(K \times r_{x}+\pi\right)+B=u_{0}$

- Assumption 3: Integrating the wind speed along the cross wind direction provides the flow mass flux. In this work, the mass flux calculated by the $2 \mathrm{D}$ wake model is considered to be equivalent to the mass flux estimated by the Jensen model

$\int_{-r_{x}}^{r_{x}}[A \cos (K \times r+\pi)+B] d r=u^{*} \times 2 r_{x}$

where $u^{*}$ is the velocity predicted by the original Jensen wake 
model. By solving a system of Eqs. (6)-(8), we get the values for the determined constants

$A=u_{0}-u^{*} ; \quad K=\pi / r_{x} ; \quad B=u^{*}$

Substituting the obtained constants into Eq. (5), the modified wind velocity $u$ can be written as

$u=\left(u_{0}-u^{*}\right) \cos \left(\pi / r_{x} \cdot r+\pi\right)+u^{*}$

It can be concluded that the application of the developed 2D Jensen model consists of two steps: the calculation of the wake velocity using original Jensen wake model, which is regarded as the first step; and then the redistribution of the velocity deficit along the cross-stream direction with a cosine shape function, which is considered as the second step. Therefore, based on the proposed 2D Jensen model wind speed in the wake region can be calculated using the following formula:

First step : $u^{*}=u_{0}\left[1-2 a /\left(1+k x / r_{1}\right)^{2}\right]$

Second step : $u=\left(u_{0}-u^{*}\right) \cos \left(\pi / r_{x} \cdot r+\pi\right)+u^{*}$

\subsection{Wake turbulence model}

To use the Jensen wake model, the critical problem is how to determine the wake decay constant. To determine the wake decay constant, an empirical Eq. (4) is used. However, in (Ishihara et al., 2004; Mittal et al., 2011; Politis et al., 2011) it is demonstrated that this equation is not so reliable because only the ambient turbulence level is considered. In fact, the wake decay is influenced by a lot of factors. In addition to the ambient turbulence level, the turbine-induced turbulence can also influence the decay constant. In Ishihara et al. (2004), Katic et al. (1986), Politis et al. (2011), through numerical tests, the wake recovery in the wake flow area is found to be underestimated if the wake decay constant in Eq. (4) is used. This can be explained by the fact that the mixing process is increased as the turbulence level is increased with turbine added turbulence (including the turbulence generated by the shear and the rotor in the wake), further leading to a faster wake recovery. Similarly, Bastankhah and Porte-Agel (2014) also point out that the wake decay coefficient is increased in the wake as the induced vortices increase turbulence intensity in the wake. In addition, experiments in Ishihara et al. (2004) have shown that for onshore sites, the rate of the wake recovery is high due to the existence of high ambient turbulence levels while for offshore sites, the wake recovery rate is more dependent on the turbine-generated turbulence since the ambient turbulence level is relatively low. All these observations indicate that the wake decay constant should be related to the effective wake turbulence composed of ambient turbulence and turbine added turbulence.

Before modifying the wake decay constant, an accurate prediction of the wake turbulence is required. Several models have been proposed to estimate the added turbulence intensity. Among others, based on both experimental and numerical approaches, Crespo and Hernandez (1996) suggest the following empirical expression for the added turbulence $I_{+}$produced by the action of the rotor in a free stream flow

$I_{+}=0.73 a^{0.8325} I_{0}{ }^{0.0325}(x / D)^{-0.32}$

where $I_{0}$ is the turbulence intensity of the incoming wind flow, $a$ is the axial induction factor (see Eq. (2)), $x / D$ is the dimensionless streamwise distance with $\mathrm{D}$ being the rotor diameter. Then, the turbulence intensity in the wake is assumed as

$I_{\text {wake }}=\sqrt{I_{0}^{2}+I_{+}^{2}}$
It should be noted here that this model is only applicable for the parameter ranges $5<x / D<15, \quad 0.07<I_{0}<0.14$ and $0.1<a<0.4$.

Frandsen et al. (1996) suggest another model for the effective turbulence intensity in the wake

$I_{\text {wake }}=\sqrt{K_{n} \frac{C_{T}}{(x / D)^{2}}+I_{0}^{2}}$

where the constant $K_{n}$ is assumed to be 0.4 for a large wind farm. Comparing Eq. (12) with Eq. (13), it is easily found that the wake turbulence intensity is suggested to be related to the free-stream turbulence level $I_{0}$, the downstream distance $x$ and the thrust coefficient of the wind turbine $C_{T}$. Although the first model uses the axial induction factor $a$ instead of the thrust coefficient, these two parameters are correlated with each other as shown in Eq. (2).

Similar to the work in (Frandsen et al., 1996), a new formula for wake turbulence intensity is proposed in this work with the following form

$I_{\text {wake }}=K_{n} \frac{C_{T}}{\chi / D}+I_{0}$

When comparing this formula with Eq. (13), it is found that the added turbulence intensity in the later is proportional to the square root of the thrust coefficient while in the proposed model the added turbulence intensity is directly proportional to the thrust coefficient. This enhances the effect of the thrust force on the wake flow development. The idea of this enhancement is based on the fact that the thrust force comes from a change in pressure as the wind passes the rotor and slows down, it is a measure of the power losses caused by the presence of the turbine, and furthermore a measure of the flow development in the wake area.

It should be noted here that the proposed wake turbulence intensity prediction model (as seen in Eq. (14)) is kind of an empirical engineering model rather than a physical model, so there is no physical meaning behind the empirical relation.

To assess the above-mentioned wake turbulence models, they are applied in cases with different types of wind turbines operating at different conditions, which are given in Table 1. The first two test cases are based on the meteorological mast measurements from the Sexbierum wind farm (Cleijne, 1993) and the Nibe site (Taylor, 1990). The last two cases (Laan et al., 2014; Wu and Porte Agel, 2012) are based on the high fidelity LES model with the aim to investigate the influence of the atmospheric turbulence intensity on wind turbine wakes. It should be pointed out here that the parameter $H$ in Table 1 is the hub height of the wind turbine, while the meanings of the other parameters have been mentioned above.

Fig. 1 presents the downstream development of the wake turbulence intensity obtained by the different models described above and some comparisons with related experimental data and LES simulation results. The LES simulations (Laan et al., 2014; Wu and Porte Agel, 2012) were combined with the actuator disc method for modeling the wind turbine rotor under the conditions listed in Table 1. To achieve fair comparisons, the corresponding

Table 1

Summary of cases and corresponding input parameters for numerical calculations.

\begin{tabular}{lccclcc}
\hline Description & $D(\mathrm{~m})$ & $H(\mathrm{~m})$ & $C_{T}(-)$ & $u_{0}(\mathrm{~m} / \mathrm{s})$ & $I_{0}(\%)$ & $z_{0}(\mathrm{~m})$ \\
\hline $\begin{array}{c}\text { Sexbirum (Cleijne, } \\
\text { 1993) }\end{array}$ & 30 & 35 & 0.75 & 8.5 & 10.0 & 0.075 \\
$\begin{array}{c}\text { Nibe (Taylor, 1990) } \\
\text { Vestas 2 MW }\end{array}$ & 40 & 45 & 0.82 & 8.5 & 10.0 & 0.070 \\
$\quad$ (Wu and Porte & 80 & 70 & 0.80 & 9.0 & 9.4 & 0.05 \\
$\quad$ Agel, 2012) & & & & & & \\
$\begin{array}{c}\text { NREL 5 MW (Laan } \\
\quad \text { et al., 2014) }\end{array}$ & 126 & 90 & 0.79 & 8.0 & 4.0 & 0.001 \\
\hline
\end{tabular}


Exp. wake center $\boldsymbol{\Delta}$ Exp. shear layer $\multimap$ Crespo model $\because$ Frandsen model $\multimap$ Proposed model
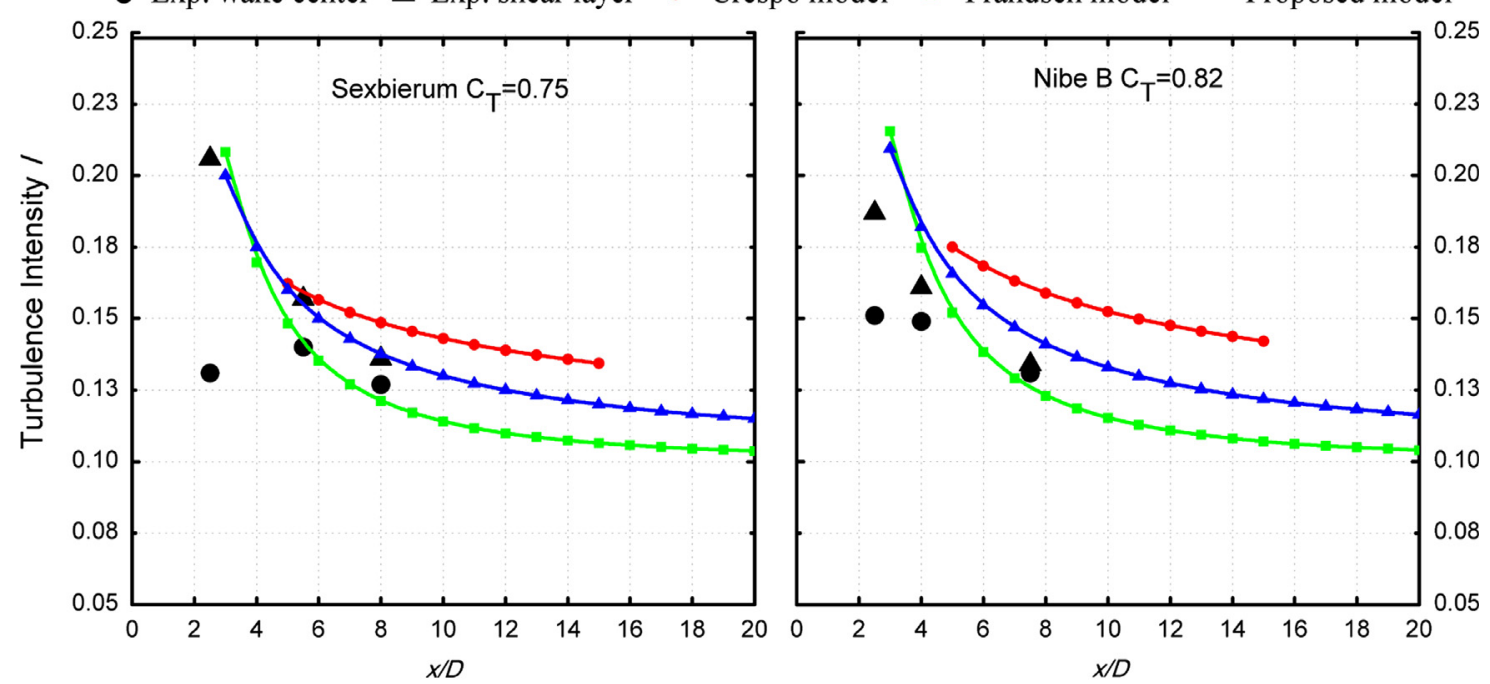

- LES. wake center $\Delta$ LES. shear layer $\rightarrow$ Crespo model $\multimap$ Frandsen model $\longrightarrow$ Proposed model
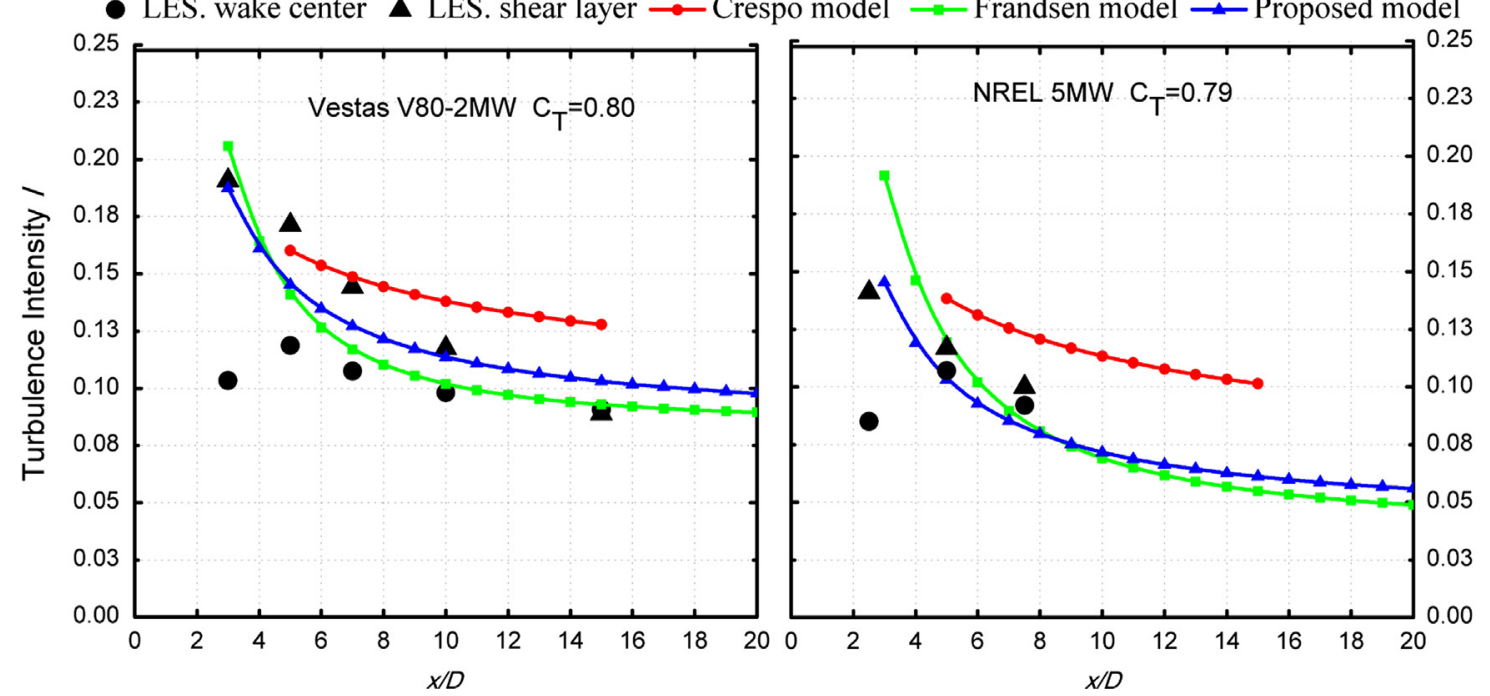

Fig. 1. Comparisons of the wake turbulence intensity calculated using Crespo model [16], Frandsen model [17] and proposed model for different types of wind turbines stated in Table 1.

measured values at the wake central-line and the peak values in the shear layer are included in Fig. 1.

Since the Crespo model is supposed to be applicable for the downstream position ranges from $5 \mathrm{D}$ to $15 \mathrm{D}$, its calculations are initiated at 5D downstream. As shown in Fig. 1, the Crespo model consistently overpredicts the turbulence intensity in the wake for all the test cases. When it comes to the Frandsen model, it can be seen that the predictions are quite close to the centerline turbulence intensity and much lower than the peak value in the shear layer. On the other hand, the calculated turbulence intensity obtained using the proposed model mainly falls between the peak and center line data and provides a reasonable prediction of the average turbulence level as compared with the measured data or LES results. This comparison demonstrates that the proposed model is worth to be used for the prediction of wake turbulence intensity in the following work.

In the wind energy projects, the wake turbulence of the upstream wind turbine has a strong impact on the fatigue and then the lifetime of the downstream turbines, thus the maximum turbulence level is an important parameter for the wind turbine design work. It should be noted that for the proposed turbulence level prediction model, as well as the Frandsen model, the limitations of them are that the average turbulence levels rather than the distributions of the turbulence level are given at given downstream position in the wake region, which means they cannot properly estimate the maximum turbulence level. But this does not imply that these models are useless for the wind turbine design process, at least they can provide an average value as a reference for the designer.

\subsection{D_k Jensen model}

Based on the estimation of the wake turbulence intensity in the above section, the modified wake decay constant can be associated with the wake turbulence through the following equation

$k_{\text {wake }}=k_{0} \frac{I_{\text {wake }}}{I_{0}}$

where $I$ is the turbulence intensity, the subscript "wake" represents parameters in the wake flow area, the subscript " 0 " means parameters in the free stream area, which can be calculated by the original Jensen model.

On the basis of the modified wake decay constant, a new wake model named as 2D_k wake model is developed where the wake decay rate $k$ is further corrected by taking into account the effective wake turbulence. The method for calculating the 
wake flow using the 2D_k Jensen model can be divided into the following steps:

- Estimate the wake turbulence intensity $I_{\text {wake }}$ at a given downstream location according to Eq. (14).

- Calculate the modified wake decay constant $k_{\text {wake }}$ using Eq. (15) with the estimated value of $I_{\text {wake }}$.

- Calculate the wake deficit using the same procedure as for the 2D Jensen model but using the modified coefficient $k_{\text {wake }}$ instead of the original wake decay constant $k$.

In order to vividly show the specific property of three different analytical wake models discussed in this article, the contour plots from the calculations predicted by these models are shown in Fig. 2. As the Jensen series models are usable to capture the velocity deficit at a downstream distance in excess of 3D, the calculations are initiated at 3D downstream.

It is observed that, in the wake flow field, the 1D Jensen model gives the uniform distribution of wake deficit in the cross wind direction while for the modified 2D Jensen models, the predicted profiles are "cosine shaped" in the cross section. Furthermore, it is clearly shown that the calculated wake recovery using the 2D_k Jensen model is faster than that given by the 2D Jensen model, and the 2D_k model also predicts a larger wake expansion than that predicted by the 2D Jensen model.

\section{Numerical tests and discussions}

In order to verify the performances of the 2D_k Jensen model, a number of numerical tests are performed on different types of wind turbines with distinct aerodynamic characters. Consequently, the accuracy of the corrected model in predicting the wake deficits of a wind turbine operating at different thrust coefficients is investigated. Additionally, the ability of the proposed models is also assessed in studying the effect of atmospheric turbulence level on

a

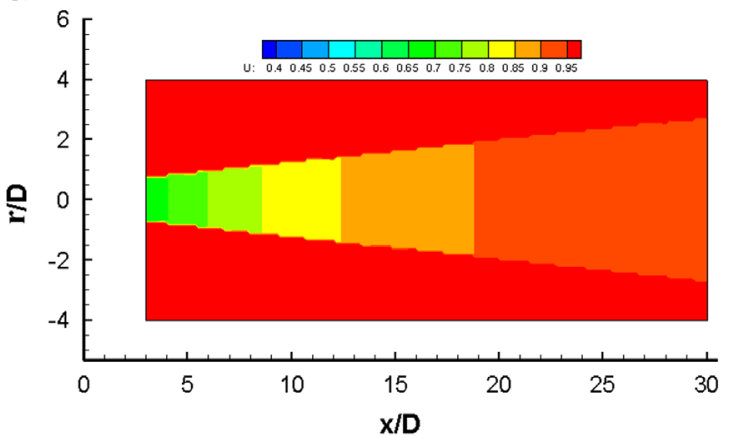

wind turbine wakes. Finally, the results obtained by the modified and standard Jensen models are compared against wind tunnel measurements, field experiments as well as some advanced CFD models.

\subsection{Case1: Sexbierum wind turbine}

The Sexbierum wind farm (Cleijne, 1993) is composed of 18 wind turbines with rated power of $310 \mathrm{~kW}$, rotor diameter of $30 \mathrm{~m}$ and hub height of $35 \mathrm{~m}$ each. The layout of 18 wind turbines positioned in this wind farm is shown in Fig. 3, in which the configuration of 7 meteorological masts (which enables the measurements of the undisturbed wind conditions for every wind direction) is also included. Measurements have been performed in the wake of T18 to investigate the wake effect, as seen in Fig. 3, through the masts contain the 3-component propeller anemometers at hub height. The measured data were analyzed based on the basis of 3-minutes averaged samples. Then the averaged velocity of $8.5 \mathrm{~m} / \mathrm{s}$ at hub height is obtained from the power

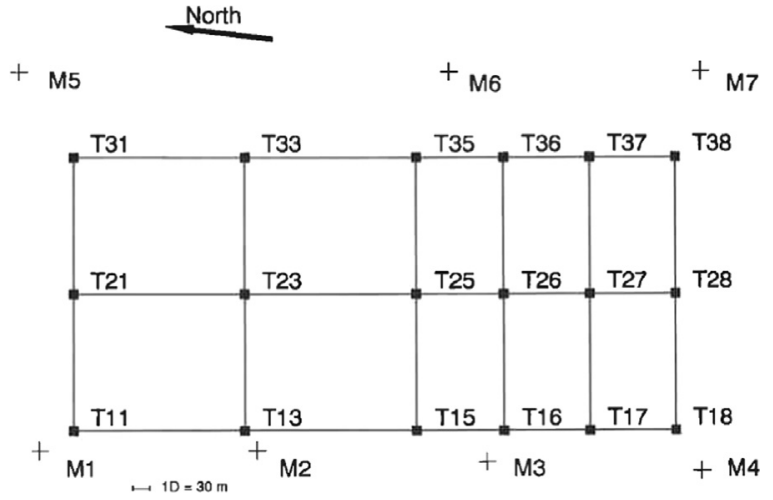

Fig. 3. Layout of Sexbierum wind farm (Cleijne, 1993).

b

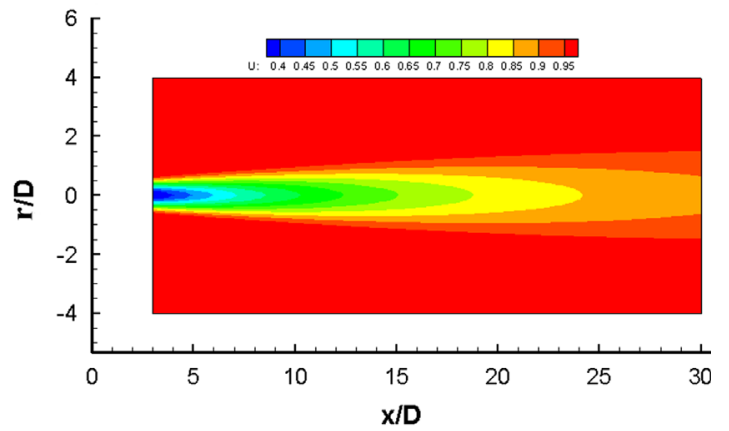

C

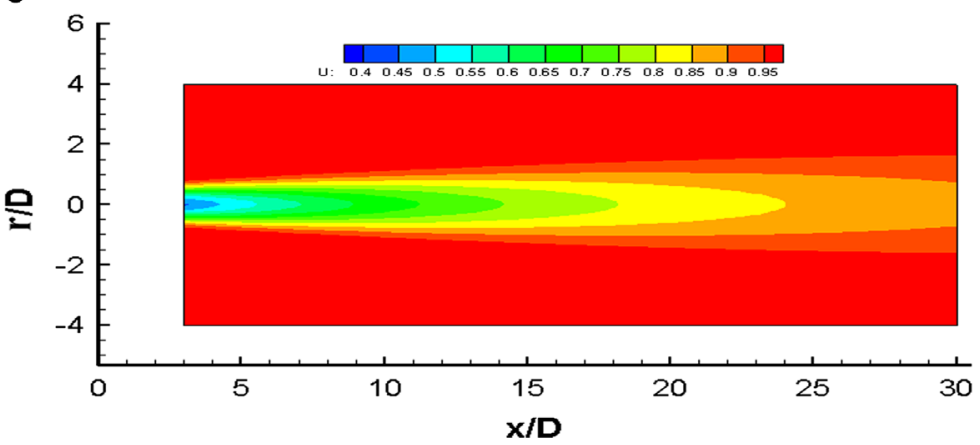

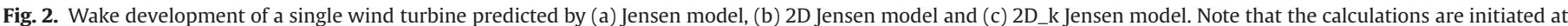
3D downstream. 

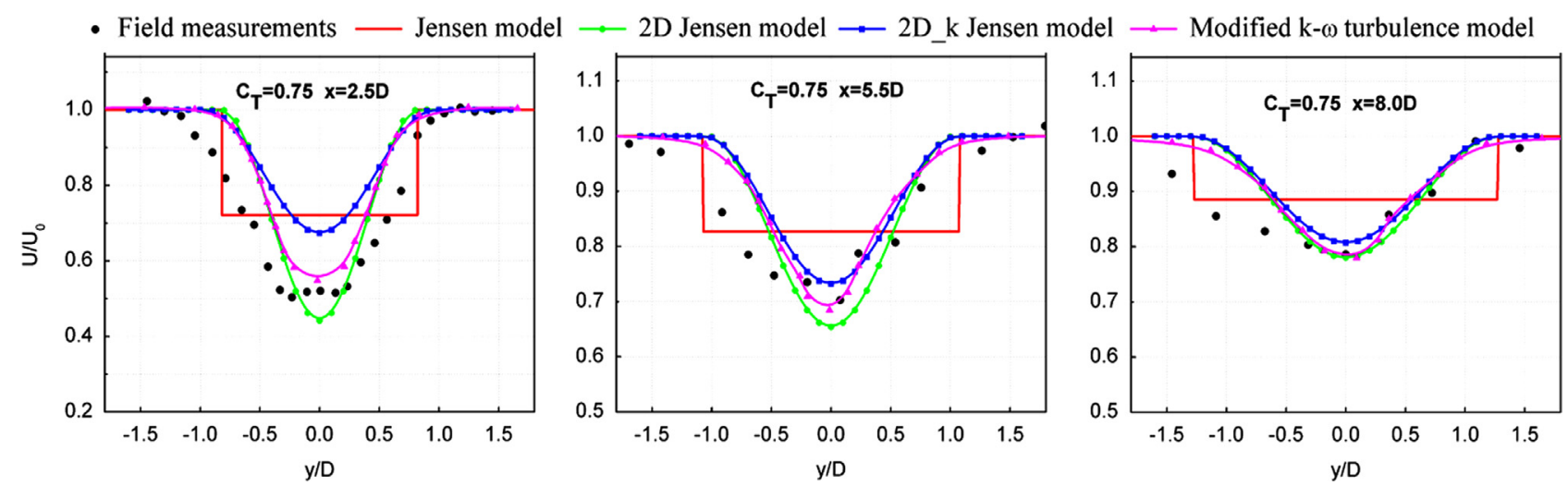

Fig. 4. Comparisons between the measured and computed wake velocity at 2.5D, 5.5D and 8.0D downstream of the turbine (a Sexbierum wind turbine with $C_{T}=0.75$ ).

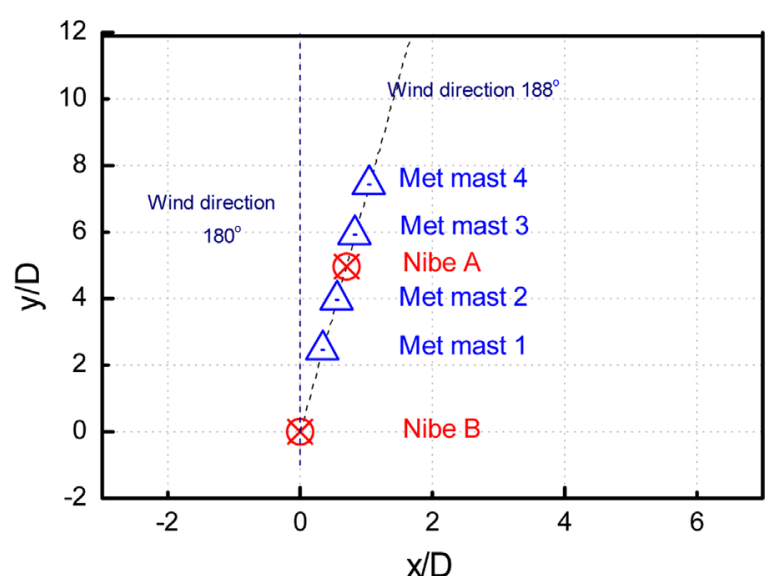

Fig. 5. Sketch of the Nibe site, including wind turbines and meteorological masts.

measurements and the known power curve. The local surface roughness of the wind farm corresponds to a turbulence intensity of $I_{0}=10 \%$ at hub height. Under these conditions, the thrust coefficient of the wind turbine is supposed to be $C_{T}=0.75$.

The comparisons of the wake speed ratio in the cross-wind direction at different downstream positions of $x / D=2.5,5.5$ and 8.0 are illustrated in Fig. 4, where the proposed 2D Jensen models are validated for the wake deficit in the near and far wake. In order to eliminate the uncertainty in the field measurements and fairly assess the Jensen series models, the numerical results obtained by the developed $k-\omega$ turbulence model (Prospathopoulos et al., 2011) are also included. This developed $k-\omega$ turbulence model was proposed through adding an additional dissipation term in the $\omega$ equation to represent the turbulence energy rate transfer from large to small scale. It should be noted that although the Jensen model are applicable in the downstream distance in excess of 3D they are still employed here to predict the near wake distribution.

It can be seen that at the position of $2.5 \mathrm{D}$, the $2 \mathrm{D}$ Jensen model compares reasonably well with the field measurements as well as the results obtained from the $k-\omega$ turbulence model, but the wake deficit predicted by the 2D_k Jensen model is quite different from the measurements. As illustrated in Fig. 4, the difference between the $2 \mathrm{D}$ Jensen model and the 2D_k Jensen model decreases as the downstream distance increases. At 5.5D, the predictions from the 2D_k Jensen model agrees very well with the measurements from the Sexbierum wind farm whereas the 2D Jensen model slightly overpredicts the wake deficit as compared with the measured one. When it comes to the position of 8.0D, the difference between the 2D Jensen model and the 2D_k Jensen model is negligible, and both models as well as the advanced $k-\omega$ turbulence model, exhibit a good agreement with the measurement.
From Eqs. (10) and (15) it can be seen that the difference between the 2D Jensen and the 2D_k Jensen models is directly influenced by the wake decay parameter $k$ which is explicitly related to the turbulence intensity. In the near wake region and the transitional area, the dominant process is the turbulence mixing from ambient, and turbine generated turbulence. So in this area, the effective turbulence level will be higher than the ambient turbulence level, which leads to the derived wake decay parameter to be larger than the wake decay constant related to the ambient turbulence intensity, results in a relatively big difference between the predicted velocity by the 2D_k and 2D Jensen models, and also results in the results from $2 \mathrm{D} \_\mathrm{K}$ Jensen model to be far from the experimental results. In the far wake (beyond 5D downstream of the wind turbine), the ambient turbulence becomes the only mixing process and the wake decay becomes similar to that in a simple diffusion problem. In this region, the wake expansion is solely determined by the ambient turbulence levels. Therefore, the wake decay parameter of the 2D_k Jensen model is quite close to that of the $2 \mathrm{D}$ Jensen model and the difference between the results of the two models is reduced or even disappeared.

\subsection{Case2: Nibe B wind turbine}

In the 1980s, filed measurements of two wind turbines at the Nibe site were conducted by Taylor (1990), the site layout is sketched in Fig. 5. The wind turbines feature a hub height and rotor diameter of $45 \mathrm{~m}$ and $40 \mathrm{~m}$, respectively. Four meteorological masts (M1, M2, M3 and M4) are placed at different downstream positions ( $x=2.5 \mathrm{D}, 4.0 \mathrm{D}, 6.0 \mathrm{D}$ and $7.5 \mathrm{D}$ ) with respect to the Nibe $\mathrm{B}$ wind turbine. One turbine named as Nibe A located 5D downstream of Nibe B was parked during the measurement period of Nibe B wind turbine. Due to the drag force of the standstill turbine, the measurements of M3 $(x=6.0 \mathrm{D})$ will not be discussed in the following analysis. All the selected data set corresponds to the Nibe B turbine operating alone were determined from the recorded longterm averaged data using an averaging period of $1 \mathrm{~min}$. The incident wind conditions at hub height were wind speed $U_{0}=8.55 \mathrm{~m} / \mathrm{s}$ (this speed was not directly measured, but estimated from the measured power and the power curve of Nibe B wind turbine) and turbulence intensity $I_{0}=10 \%$. At this condition, the thrust coefficient is estimated to be $C_{T}=0.82$.

Fig. 6 compares the computed and measured horizontal velocity at three different downstream positions, where the LES data reported in (Troldborg et al., 2014) are also included. As we can see, compared with the measurements, the wake deficit is underpredicted by the $2 \mathrm{D} \_\mathrm{k}$ Jensen model at the position of $2.5 \mathrm{D}$. As the downstream distance increases (such as $4 \mathrm{D}$ and 7.5D) the discrepancy decreases and finally a good match with the measurement is seen at the center of the wake (where the maximum velocity deficit 

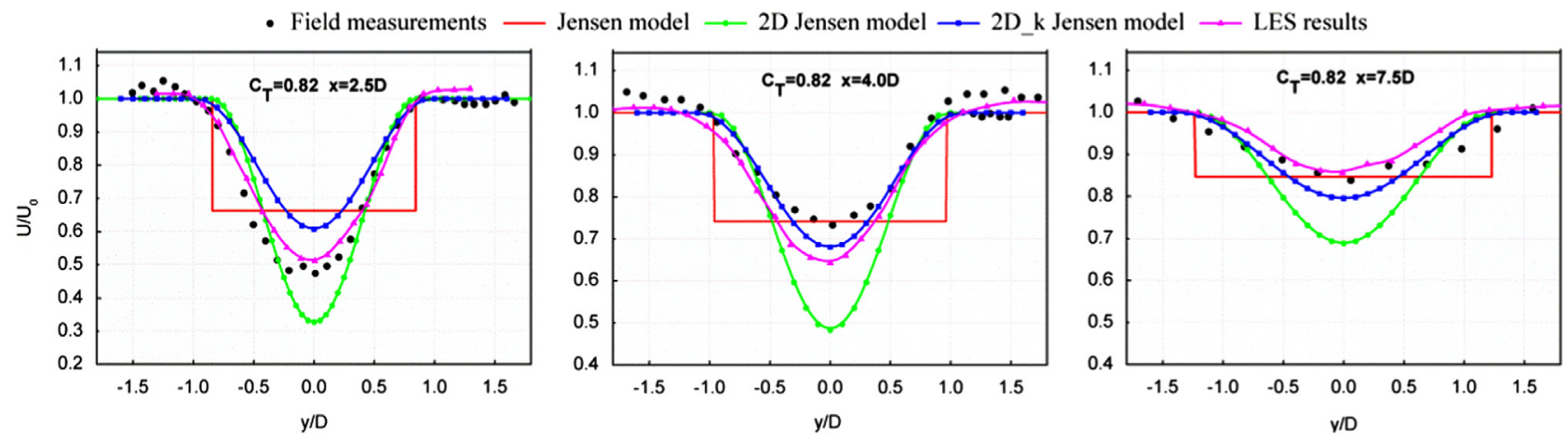

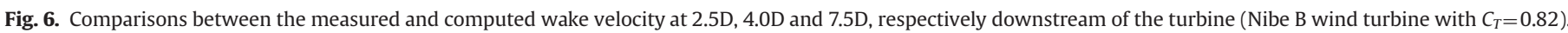

is found). Overall, for the $2 \mathrm{D} \_\mathrm{k}$ Jensen model, the agreement between the computations and measurements is generally good except for the near wake position $(x=2.5 \mathrm{D})$ where the maximum wake deficit is underpredicted by $18 \%$ as compared with the measurements. As for the 2D Jensen model, just as shown in Fig. 6, it can be seen that the wake deficit at the center of the wake is overpredicted at all downstream distances. This can be explained by the fact that the profile of the velocity deficit of the 2D Jensen model is obtained from the redistribution of the top-hat velocity profile of the original Jensen model using a cosine function, from this it can be expected that when the wake velocity predicted by Jensen model is close to the measured minimum velocity near the wake central-line (as seen in Fig. 6), the wake velocity predicted by $2 \mathrm{D}$ Jensen model will be much lower than the measurements in the wake center area.

In general, the LES data compares reasonably well with the measurements with the exception at 4.0D downstream position, but the overprediction of the wake deficit is similar to that of the $2 \mathrm{D} \_\mathrm{k}$ Jensen model. This discrepancy might be due to the fact that the measurements were affected by the turbine Nibe A which positioned at $1 \mathrm{D}$ upstream of the meteorological mast. Although Nibe A was not in operation during the measurements, it still leads to a higher value of wind speed in the vicinity of this machine because of the obstacle blocking effect.

\subsection{Case3: GH wind turbine with different thrust coefficients}

In 1989 Garrad Hassan carried out a detailed analysis of wind turbine wakes in a large atmospheric wind tunnel (Schlez et al., 2003). The analysis chose a $1 / 160$ scale wind turbine model, which corresponds to a full scale turbine with a rotor diameter of $43.2 \mathrm{~m}$ and a hub height of $50 \mathrm{~m}$. The wind turbine is supposed to be positioned in a flat terrain with a roughness length of $0.075 \mathrm{~m}$. The incoming wind speed is assumed to be $5.3 \mathrm{~m} / \mathrm{s}$, two different operating condition $C_{T}=0.62$ and $C_{T}=0.85$ are selected in our work.

The predictions obtained from the Jensen series wake models are compared with wind tunnel measurements for a single wind turbine operating at two thrust coefficients as illustrated in Fig. 7, where the cross wind profiles of mean velocity (the data were averaged over 10-minute period) at hub height, and different far wake positions are presented. For the lower thrust coefficient, it seems that the 2D Jensen wake model predicts the wake velocity rather well in terms of both shape and deficit amplitude at all the selected downstream locations. However, the agreement between the results obtained from the 2D_k model and the measured value is less favorable, and the largest error over the mean velocity is about $8 \%$ at 5D and decreases to 3\% at the further downstream of 10D. On the contrary, for the higher thrust coefficient, the results of the 2D_k Jensen model compare well with the measurements in the far wake region while the 2D Jensen model consistently overpredicts the wake deficit in the same position.
Through the comparisons of the results obtained for $C_{T}=0.62$ and 0.85 , it can be seen that the results are in accordance with the theory that a higher thrust coefficient results in more serious wake deficit than the lower one. Furthermore, the discrepancies of the 2D Jensen model and 2D_k Jensen model are smaller at the lower thrust coefficient while large discrepancies are observed for the higher thrust case. This is mainly due to the fact that the modified wake decay constant is a linear function of the thrust coefficient, and a higher thrust coefficient will be responsible for larger wake decay rate, which further results in the obvious discrepancy between the 2D Jensen model and its modification, the 2D_k Jensen model.

\subsection{Case 4: Vestas V80-2MW wind turbine with different turbulence} levels

In order to investigate the effect of atmospheric turbulence on wind turbine wakes, an LES model was employed to simulate the wake of a stand-alone wind turbine with four different aerodynamic roughness lengths (Wu and Porte Agel, 2012). The LES model, coupled with the ADM-R wind turbine model (actuator disc model with rotation) has been validated on the prediction of the wake of a single wind turbine operates in neutrally-stratified atmospheric conditions in their work, with the main purpose to investigate the effect of incoming boundary layer flow characteristics on the development of wind turbine wakes. Of these, the two cases $z_{0}=0.05$ and $z_{0}=0.005$ (which correspond to a turbulence intensity of $I_{0}=9.4 \%$ and $I_{0}=6.9 \%$ at hub height, respectively) are chosen in this work to test the ability of the proposed 2D Jensen models. In this subsection, the Vestas V80-2 MW wind turbine with a rotor diameter of $80 \mathrm{~m}$ and a hub height of $75 \mathrm{~m}$ is investigated. Based on the operational record in (Hansen et al., 2011), for the following investigations, the incoming wind speed at hub height is $U_{0}=5.3 \mathrm{~m} / \mathrm{s}$, and under this condition, the thrust coefficient is $C_{T} \approx 5.3$

Horizontal profiles of the velocity deficit at hub height at chosen downstream locations $(x / D=3,5,7,10)$ are shown in Fig. 6, in which the predictions obtained with the analytical Jensen series models and the LES data at different ambient turbulence levels are presented. It is shown that the Jensen wake model underestimates the wake deficit, relatively to the LES simulations, at the center of the wake and overestimates it near the edge of the wake at the downstream position of $3 \mathrm{D}$, and the difference between the Jensen model predictions and the LES data decreases as the downstream distance increases. For the performance of the 2D Jensen model, it is clearly seen that this model overpredicts the wake deficit consistently, relatively to the LES simulations, in the whole wake flow area, with the largest discrepancy occurring at the wake center of the 3.0D case, up to about $23 \%$ for $z_{0}=0.05$ and to $40 \%$ for $z_{0}=0.005$. In contrast, the comparisons show that the 2D_k Jensen model predicts the wake velocity more accurately than the other two models and exhibits an acceptable agreement with the wake deficit predicted by LES in both conditions. It needs 

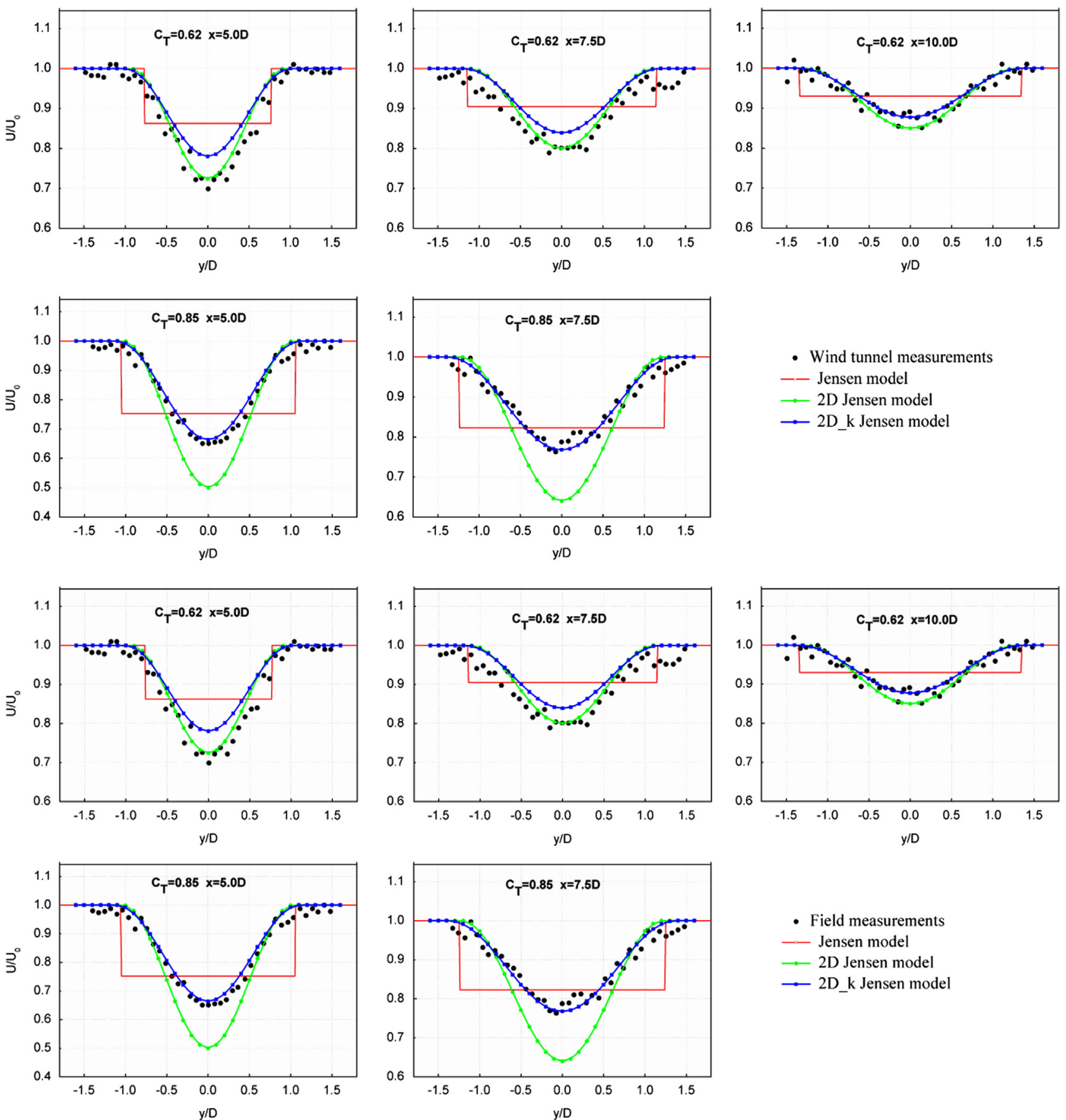

- Field measurements

Jensen model

$\because 2 \mathrm{D}$ Jensen model

$\because$ 2D_k Jensen model

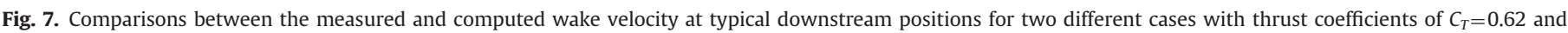
$C_{T}=0.85$, respectively.

to be mentioned here that the LES results are numerical calculation results instead of the measurements, so from this point of view it seems this LES test case cannot convincingly prove the accuracy of the 2D_k Jensen model, but at least this test shows that the predictions of the analytical wake model are much close to the results of the advanced CFD model. Fig. 8.

It is known that a higher turbulence leads to relatively weaker wake effect and relatively faster wake recovery than a lower one. This is mainly due to the fact that the higher ambient turbulence enhances the wake mixing process which produces more turbinegenerated turbulence contributing to the decay rate of wind turbine wake, and results in a faster wake recovery. As expected, from the results for case $z_{0}=0.05$ and case $z_{0}=0.005$, it is found that the proposed models seem to show this as well.

\section{Conclusions and further work}

In this study, based on the Jensen model, two new analytical models are proposed for predicting the wake deficit for single wind turbines. To overcome the shortage of the Jensen model, a more physical model named as 2D Jensen model is proposed with the main idea of employing a cosine distribution instead of the top-hat shape to represent the cross-stream profile of wake deficit. Particularly, a 2D_k Jensen model is developed based on the 2D Jensen model with further modification of the wake decay constant. For the 2D_k Jensen model, the effect of ambient turbulence level as well as the rotor generated turbulence is taken into account which leads to a new wake decay constant compared to a fixed wake decay constant used in the original Jensen model. Besides, a suitable 

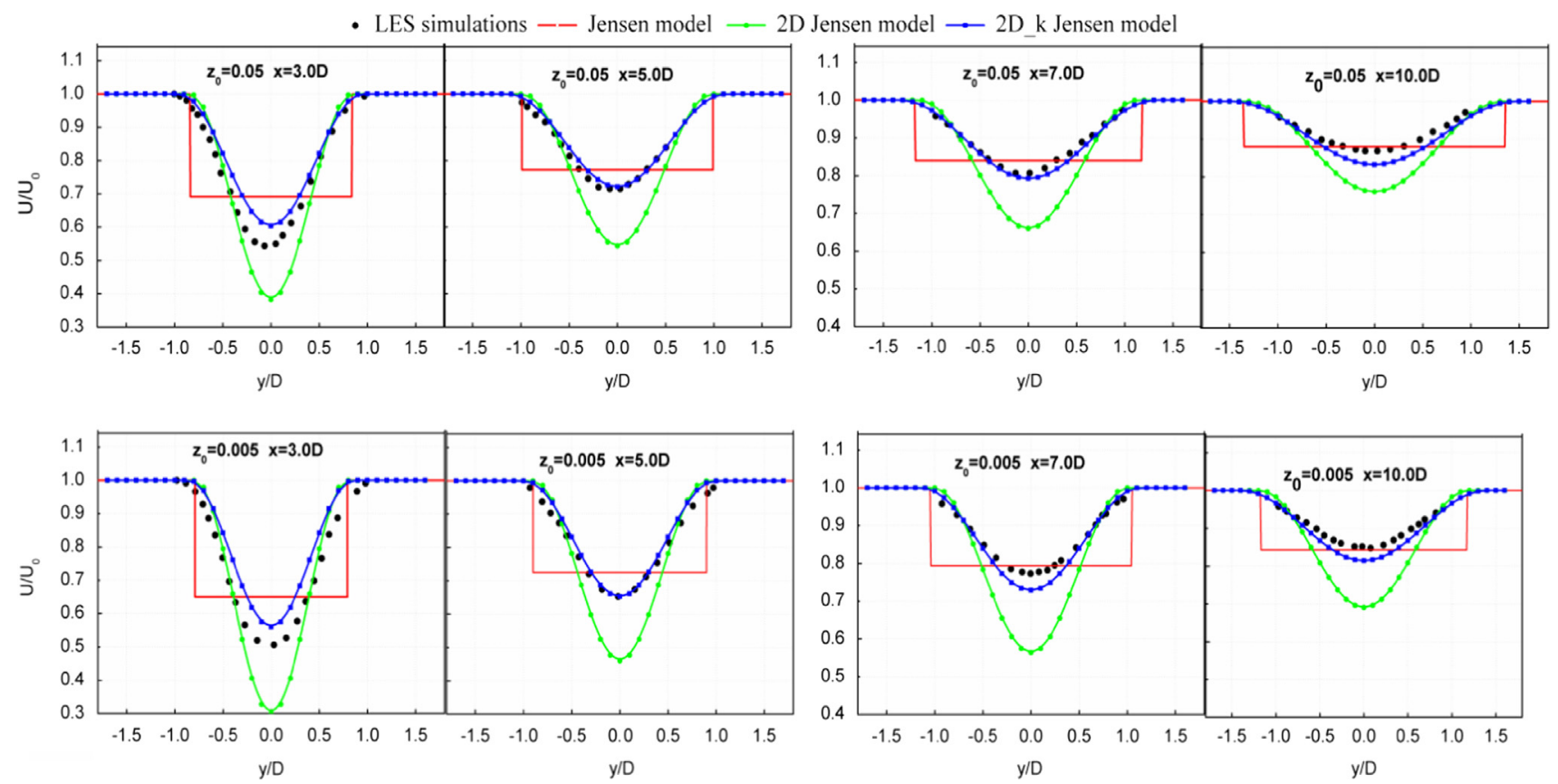

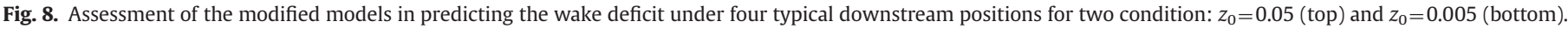

model for average estimation of the turbulence intensity in the wake flow is also included in the 2D_k Jensen model.

The comparisons of the analytical models with field measurements, wind tunnel experiments and an advanced CFD model show that the velocity profile in the wake obtained with the proposed model (2D_k Jensen model) is in acceptable agreement with the experimental and CFD results in the above discussed numerical test cases where different types of wind turbines under various atmospheric conditions are studied. The comparisons reveal also that for a lower thrust coefficient (such as the GH case), the 2D Jensen model shows more comparable results with respect to the measurements while the 2D_k Jensen model slightly underestimates the wake deficit effect with the maximum error of $8 \%$ at the center of the wake at the position $x=5.0 \mathrm{D}$ and the error declines in the further downstream. But for other cases such as the cases with higher thrust coefficient or with higher turbulence levels, the 2D_k Jensen model is consistently and acceptably accurate in terms of both shape of deficit and amplitude of the mean velocity wake in the far wake area. With respect to the near wake region, the 2D Jensen model is generally more accurate than the 2D_k Jensen model. But in most cases of practical interest the typical intermaching spacing falls in the range of $5 \mathrm{D}$ to $15 \mathrm{D}$. It seems that the $2 \mathrm{D} \_\mathrm{k}$ Jensen model is capable of proving reasonable estimations of the wake deficit over this region of most interest.

Based on the various studies in this article, it is concluded that the proposed 2D_k Jensen model is a suitable tool for predicting the effective turbulence and the velocity in the wake of a single wind turbine. It is expected that the 2D_k Jensen model will be extended to evaluate the power output of wind farms for different layout configurations and inflow wind conditions in future studies.

\section{Acknowledgments}

This work is supported by the National Basic Research Program of China (the "973” program. 2014CB046200), NSFC (grant 91130030) and the Danish Research Council for Strategic Research (DSF sagsnr. 10-094544).

\section{References}

Barthelmie, R.J., Folkerts, L., Larsen, G.C., et al., 2006. Comparison of wake mode simulations with offshore wind turbine wake profiles measured by sodar. J. Atmos. Ocean. Technol. 23 (7), 888-901.

Barthelmie, R.J., Hansen, K., Frandsen, S.T., et al., 2009. Modelling and measuring flow and wind turbine wakes in large wind farms offshore. Wind Energy 12 (5), 431-444.

Barthelmie, R.J., Pryor, S.C., Frandsen, S.T., et al., 2010. Quantifying the impact of wind turbine wakes on power output at offshore wind farms. J. Atmos. Ocean. Technol. 27 (8), 1302-1317.

Bastankhah, M., Porte-Agel, F., 2014. A new analytical model for wind-turbine wakes. Renew. Energy 70 (1), 116-123.

Chamorro, L.P., Porte-Agel, F., 2009. A wind-tunnel investigation of wind-turbine wakes: boundary-layer turbulence effects. Bound.-Layer Meteorol. 132 (1), $129-149$.

Cleijne, J., 1993. Results of the Sexbierum Wind Farm: Single Wake Measurements. TNO Report.

Crespo, A., Hernandez, J., 1996. Turbulence characteristics in wind-turbine wakes. J. Wind Eng. Ind. Aerodyn. 61 (1), 71-85.

Crespo, A., Hernandez, J., Frandsen, S., 1999. Survey of modelling methods for wind turbine wakes and wind farms. Wind Energy 2 (1), 1-24.

Dufresne, N.P., Wosnik, M., 2013. Velocity deficit and swirl in the turbulent wake of a wind turbine. Mar. Technol. Soc. J. 47 (4), 193-205.

Frandsen, S.T., Chacón, L., Crespo, A. et al., 1996. Measurements on and modelling of offshore wind farms. 1996, Risø National Laboratory, Risø-R-903 (EN).

Hansen, K.S., Barthelmie, R.J., Jensen, L.E., et al., 2011. The impact of turbulence intensity and atmospheric stability on power deficits due to wind turbine wakes at Horns Rev wind farm. Wind Energy 15 (1), 183-196.

Ishihara, T., Yamaguchi, A., Fujino, Y., 2004. Developement of a new wake model based on a wind tunnel experiment. Glob. Wind Power. http://windeng.t. u-tokyo.ac.jp/ishihara/e/.

Jensen, N.O., 1983. A Note on Wind Generator Interaction. Risø National Laboratory, Roskilde, Denmark.

Katic, I., Højstrup, J., Jensen, N., 1986. A simple model for cluster efficiency. Eur. Wind Energy Assoc. Conf. Exhib. 1, 407-410.

Laan, M.P., Sørensen, N.N., Réthoré, P.E., et al., 2014. An improved k- $\epsilon$ model applied to a wind turbine wake in atmospheric turbulence. Wind Energy, http: //dx.doi.org/ 10.1002/we.1736.

Magnusson, M., Smedman, A.S., 1999. Air flow behind wind turbines. J. Wind Eng. Ind. Aerodyn. 80 (1-2), 169-189.

Mittal, A., Taylor, L.K., Sreenivas, K. et al., 2011. Investigation of two analytical wake models using data from wind farms. ASME 2011 International Mechanical Engineering Congress and Exposition. IMECE 2011, United states, pp. 1215-1222.

Politis, E.S., Prospathopoulos, J., Cabezon, D., et al., 2011. Modeling wake effects in large wind farms in complex terrain: the problem, the methods and the issues. Wind Energy 15 (1), 161-182. 
Porte Agel, F., Wu, Y.T., Chen, C.H., 2013. A numerical study of the effects of wind direction on turbine wakes and power losses in a large wind farm. Energies 6 (10), 5297-5313.

Prospathopoulos, J.M., Politis, E.S., Rados, K.G., et al., 2011. Evaluation of the effects of turbulence model enhancements on wind turbine wake predictions. Wind Energy 14 (2), 285-300.

RÉTHORÉ, P.-E., 2006. Thrust and Wake of a Wind Turbine: Relationship and Measurements ([Master degree]). Technical University of Denmark, Risø National Laboratory, Roskilde, Denmark.

Schlez, W., Tindal, A., Quarton, D.,, 2003. GH Wind Farmer Validation Report. Garrad Hassan and Partners Ltd, Bristol.

Taylor, G. Wake Measurements on the Nibe Wind Turbines in Denmark. National Power, ETSU WN 5020, United Kindom, 1990.
Troldborg, N., Sørensen, J.N., Mikkelsen, R., et al., 2014. A simple atmospheric boundary layer model applied to large eddy simulations of wind turbine wakes. Wind Energy 17 (4), 657-669.

Vermeer, L.J., Sørensen, J.N., Crespo, A., 2003. Wind turbine wake aerodynamics. Prog. Aerosp. Sci. 39 (6-7), 467-510.

Wu, Y.T., Porte Agel, F., 2012. Atmospheric turbulence effects on wind-turbine wakes: an LES study. Energies 5 (12), 5340-5362. 